\title{
Pathogens isolated from clinical mastitis in Murrah buffaloes and their antibiogram
}

\author{
Gaurav Charaya ${ }^{1}$, Anshu Sharma², Ashok Kumar ${ }^{1}$, Mahavir Singh² and Parveen Goel ${ }^{1}$
}

1. Department of Veterinary Medicine, College of Veterinary Sciences, Lala Lajpat Rai University of Veterinary and Animal Sciences, Hisar, Haryana, India; 2. College Central Laboratory, College of Veterinary Sciences, Lala Lajpat Rai University of Veterinary and Animal Sciences, Hisar, Haryana, India.

Corresponding author: Anshu Sharma, e-mail: anshusharma_dr@yahoo.com, GC: gcharaya9@gmail.com, AK: aggar2050@hotmail.com, MS: drmahaviryadav@gmail.com, PG: parveengoel.hsr@gmail.com

Received: 21-07-2014, Revised: 14-10-2014, Accepted: 23-10-2014, Published online: 27-11-2014

doi: 10.14202/vetworld.2014.980-985. How to cite this article: Charaya G, Sharma A, Kumar A, Singh M, Goel P (2014) Pathogens isolated from clinical mastitis in Murrah buffaloes and their antibiogram, Veterinary World 7(11): $980-985$.

\begin{abstract}
Aim: To determine the etiology of clinical mastitis in Murrah buffaloes and to develop an antibiogram of organisms isolated.

Materials and Methods: A total of 564 quarter milk samples of 144 buffaloes suffering from clinical mastitis received in Veterinary College Central Laboratory were inoculated on blood agar, MacConkey's lactose agar and sabouraud dextrose agar. Bacteria isolated were characterized to the species level and subjected to in-vitro antimicrobial sensitivity testing.

Results: Out of 564 quarters examined for mastitis, 320 (56.73\%) quarters were found culturally positive showing isolation of Staphylococcus aureus 140 (38.04\%), Streptococcus dysgalactiae 112 (30.43\%), Streptococcus agalactiae 13 (3.53\%), Escherichia coli 74 (20.10\%) and Corynebacterium pyogenes 29 (7.88\%). On carrying antibiogram staphylococci and streptococci revealed high sensitivity towards chloramphenicol, gentamicin, amikacin and enrofloxacin. Streptococci showed high sensitivity towards ceftriaxone and cefaperazone also. E. coli was found highly sensitive to chloramphenicol and gentamicin. C. pyogenes was sensitive to the majority of antibiotics.
\end{abstract}

Conclusion: S. aureus was the most predominant bacteria isolated from mastitis cases and irrespective of the isolates chloramphenicol was found to be most sensitive when tested in-vitro.

Keywords: antibiogram, Escherichia coli., mastitis, staphylococci, streptococci.

\section{I ntroduction}

India ranks first in milk production with 121.8 million tonnes of milk production [1] of which more than 55\% comes from buffaloes. Haryana has the world's best dairy type buffalo, the Murrah, capable of milk yields as high as $35 \mathrm{~kg}$ a day. Mastitis is an inflammation of mammary glands causing heavy economic losses to the dairy industry throughout the world. These losses in India have been increased from Rs. 6053.21 crores [2] to Rs. 7165.51 crores per annum [3].

At present it has been established that mastitis can be caused by over 250 microorganisms [4] and detection of these pathogens is considered essential for the definitive diagnosis of mastitis. Treatment with antibiotics is one of the most important components to control mastitis. Due to indiscriminate use of antibiotics, antibiotic resistance has been developed against most of the commonly used drugs leading to treatment failure. Therefore, definitive diagnosis of etiological agents and their antimicrobial sensitivity prior to treatment will help in selecting suitable and cost effective antibiotic to treat an animal properly.

Therefore, the present study was planned to determine the etiological agent(s) responsible for causation of clinical mastitis in Murrah buffaloes

Copyright: The authors. This article is an open access article licensed under the terms of the Creative Commons Attributin License (http:// creative commons.org/licenses/by/2.0) which permits unrestricted use, distribution and reproduction in any medium, provided the work is properly cited. and to determine their antimicrobial sensitivity to institute proper line treatment and adoption of control measures.

\section{Materials and Methods}

Ethical approval

State project on mastitis is under operation in College Central Laboratory, Lala Lajpat Rai University of Veterinary and Animal Sciences (LUVAS), Hisar. The milk samples used in the current study were directly received from animal owners. As per University rules for these samples approval of Institutional Animal Ethics Committee is not required.

\section{Place of study}

The study was conducted in College Central Laboratory, College of Veterinary Sciences, LUVAS, Hisar.

\section{Source of milk samples}

A total of 564 quarter milk samples were received directly from animal owners of 144 buffaloes suffering from clinical mastitis in a sterile container from different parts of Haryana and nearby villages of Rajasthan.

\section{Bacteriological examination}

Immediately after receiving, the milk samples were inoculated in $0.01 \mathrm{ml}$ volume on 5\% sheep blood agar (BA), MacConkey’s lactose agar (MLA) and sabouraud dextrose agar plates with the help of a $4 \mathrm{~mm}$ diameter platinum loop. The plates 
were incubated aerobically at $37^{\circ} \mathrm{C}$ for $24-48 \mathrm{~h}$. Sub-cultures of the resulting growth were made on BA for purification of isolates and identified on the basis of Grams reaction, morphology and colony characteristics. All the isolates were characterized up to species' level following standard microbial procedures of National Mastitis Council [5].

The Gram-positive cocci were subjected to catalase test to differentiate between staphylococci and streptococci. To rule out possibility of micrococci, all the catalase positive cocci were subjected to oxidase test and oxidation fermentation test. On the basis of coagulase test, haemolysis on 5\% BA and Staph ${ }^{\mathrm{TM}}$ latex test kit (HiMedia, Mumbai), staphylococci were identified up to species' level.

The organisms that on preliminary examination were found to be streptococci, were further characterized for different species using Strep ${ }^{\mathrm{TM}}$ latex test kit (HiMedia, Mumbai, India) as per instructions of the manufacturer. Other organisms like Corynebacterium pyogenes were identified on the basis of colony characteristics, Grams staining reaction, morphology and catalase test, whereas Escherichia coli were differentiated on the basis of growth on eosin methylene blue agar, MLA and IMViC test.

\section{Antimicrobial susceptibility testing}

Different strains of various organisms isolated from udder infections were subjected to in-vitro drug sensitivity testing, using 14 antimicrobials by disc-diffusion method [6]. With the help of a platinum loop, a small amount of test culture was transferred into a tube of brain heart infusion broth and incubated for $2-5 \mathrm{~h}$ at $37^{\circ} \mathrm{C}$, so as to obtain turbidity. With the help of a sterile cotton swab, the broth culture was then evenly spread by smearing over the surface of BA/Mueller-Hinton agar plates. The antimicrobial discs were placed on the agar and gently pressed. These were then, incubated at $37^{\circ} \mathrm{C}$ for $24 \mathrm{~h}$. The sensitivity was observed on the basis of zone size interpretation chart, provided by the manufacturer. The results were recorded as sensitive, intermediate and resistant. Different antimicrobials used were ampicillin (25 mcg), ceftriaxone (30 mcg), amoxicillin (10 mcg), streptomycin (25 mcg), cloxacillin (30 mcg), chloramphenicol (30 mcg), neomycin (30 mcg), tylosin (15 mcg), enrofloxacin (10 mcg), cefoperazone (75 mcg), amikacin (30 mcg), penicillin-G (10 units), gentamicin (30 mcg), and oxytetracycline (30 mcg) (Hi media).

\section{Result}

Prevalence of mastitis on the basis of bacteriological examination was found to be $56.73 \%$ quarter wise. Out of 564 quarters examined for mastitis, 320 quarters were found culturally positive of which 48 quarters were showing mixed dual bacterial infection, therefore accounting for total 368 isolates $(320+48)$. These organisms were identified as
Staphylococcus aureus 140 (38.04\%), Streptococcus dysgalactiae 112 (30.43\%), Streptococcus agalactiae 13 (3.53\%), E. coli 74 (20.10\%) and C. pyogenes 29 (7.88\%). None of the milk samples showed growth of fungi. The frequency of isolation of different bacterial species is depicted in Table-1.

The results of antimicrobial sensitivity testing have been shown in Table-2. Staphylococci (140) revealed antibiotic sensitivity in descending order to amikacin (88.57\%), gentamicin (86.42\%), chloramphenicol (84.28\%), enrofloxacin (82.14\%), cefoperazone (81.42\%), ceftriaxone and streptomycin (79.28\%), oxytetracycline (77.14\%), tylosin (72.85\%), ampicillin (67.85\%), cloxacillin (63.57\%), neomycin (61.42\%), amoxicillin (52.14\%) and least towards penicillin-G (29.28\%).

In case of streptococci (125) irrespective of species the maximum sensitivity $97.00 \%$ was seen towards chloramphenicol followed by ceftriaxone, cefoperazone, and enrofloxacin (95.20\%), amikacin, gentamicin and ampicillin (92.00\%), oxytetracycline (87.20\%), tylosin and neomycin (84.80\%), streptomycin (82.40\%), amoxicillin (72.00\%), cloxacillin (69.00\%) and penicillin-G (56.00\%).

Variable sensitivity was shown by E. coli (74) isolates with maximum sensitivity towards chloramphenicol (94.59\%) and least towards cloxacillin (8.10\%). In the present study sensitivity of E. coli isolates towards gentamicin, ceftriaxone, enrofloxacin and amikacin were in the range of $74.32-82.43 \%$. However, sensitivity to other antibiotics was comparatively low. Only $17.56 \%$ isolates were sensitive to tylosin.

All 29 (100\%) isolates of $C$. pyogenes were sensitive to streptomycin, chloramphenicol, cloxacillin, amikacin, gentamicin, amoxicillin, enrofloxacin, ceftriaxone and cefoperazone whereas $89.65 \%$ were sensitive for ampicillin, neomycin and tylosin. Least sensitivity was shown towards oxytetracycline (77.41\%) and penicillin-G (68.96\%).

Table-1: Relative frequency of organisms isolated from 564 milk samples of 144 buffaloes.

\begin{tabular}{lcc}
\hline Parameters & Buffalo (144) & Percentage \\
\hline Total quarters & 576 & - \\
Quarters examined & 564 & - \\
Quarters culturally positive & 320 & 56.73 \\
Quarters culturally negative & 244 & 43.27 \\
Total isolates & 368 & - \\
Staphylococci & 140 & 38.04 \\
Streptococci & 125 & 33.96 \\
E. coli & 74 & 20.10 \\
C. pyogenes & 29 & 7.88 \\
Mixed infections & $48^{*}$ & 15.00 \\
\hline
\end{tabular}

*Staphylococci+Streptococci (17),

Streptococci+C. pyogenes (6), Staphylococci+E. coli (13), Staphylococci+C. pyogenes (6), Streptococci+E. coli (6),

C. pyogenes $=$ Corynebacterium pyogenes,

E. coli=Escherichia coli 
Table-2: Antibiogram pattern of isolates obtained from 564 quarters milk samples.

\begin{tabular}{|c|c|c|c|c|c|c|c|c|c|c|c|c|c|c|}
\hline Organism (isolates) & $\mathbf{P}$ & $\mathbf{S}$ & $\mathbf{0}$ & C & A & $\mathbf{N}$ & Cx & Ex & G & Ak & Am & Ctr & Cf & TI \\
\hline 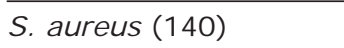 & 29.28 & 79.28 & 77.14 & 84.28 & 67.85 & 61.42 & 57 & 82.14 & 86 & 7 & 52.14 & 28 & 42 & 12.00 \\
\hline 5) & 56.00 & 82.40 & 87.20 & 97.00 & 92.00 & 84.80 & 69.00 & 95.20 & 92.00 & & 72.00 & 95 & & 84.80 \\
\hline 74) & 13.51 & 68.91 & 60.81 & 94.59 & 27.02 & 35.13 & 8.10 & 78.57 & 82.43 & 78.37 & 17.56 & 74.32 & 60.81 & 17.56 \\
\hline acter & 68.96 & 100 & 79.31 & 100 & 89.65 & 89.65 & 100 & 100 & 100 & 100 & 100 & 100 & 100 & 89.65 \\
\hline Total (368) & 38.91 & 19.88 & 77.44 & 91.91 & 69.56 & 66.29 & 57.13 & 87.22 & 88.58 & 88.58 & 55.70 & 85.32 & 83.42 & 67.11 \\
\hline
\end{tabular}

S. aureus=Staphylococcus aureus, E. coli=Escherichia coli, A=Ampicillin ( $25 \mathrm{mcg}), \mathrm{Ctr}=$ Ceftriaxone $(30 \mathrm{mcg})$, Am=Amoxycillin $(10 \mathrm{mcg}), \mathrm{S}=$ Streptomycin $(25 \mathrm{mcg}), \mathrm{Cx}=$ Cloxacillin $(30 \mathrm{mcg}), \mathrm{C}=$ Chloramphenicol $(30 \mathrm{mcg})$, $\mathrm{N}=$ Neomycin $(30 \mathrm{mcg}), \mathrm{Tl}=$ Tylosin $(15 \mathrm{mcg}), \mathrm{Ex}=$ Enrofloxacin $(10 \mathrm{mcg}), \mathrm{Cf}=$ Cefoperazone $(75 \mathrm{mcg}), \mathrm{Ak}=\mathrm{Amikacin}$ (30 mcg), P=Penicillin-G (10 units), G=Gentamicin (30 mcg), and $\mathrm{O}=0 x y$ tetracycline ( $30 \mathrm{mcg}$ )

\section{Discussion}

\section{Prevalence of different microorganisms}

Among the various microorganisms isolated, S. aureus was the most prevalent followed by Streptococcus spp., which was in accordance with reports of several workers [7-13]. Sumathi et al. [14] also reported staphylococci as the most important and prevalent mastitis causing organism globally, including India. High prevalence of $S$. aureus points to poor milking time hygiene as this pathogen is mainly spread during and between two milking via milkers' hands and towels. S. aureus is also of public health importance as enterotoxigenic strains can cause food poisoning in human beings. Moreover, the existence of high concentration of $S$. aureus in milk also indicates the relatively poor quality of milk and milk products. These results also show that in India among streptococci, S. agalactiae and S. dysgalactiae are the most frequently encountered organisms followed by other species of streptococci. In developed countries like USA and UK, a dramatic decrease in the incidence of contagious mastitis pathogens has been achieved by implementing five-point mastitis control programme. In these countries infections caused by the $S$. agalactiae and $S$. dysgalactiae have been eradicated or reduced to very low levels, and the effect on staphylococcal infection is similar though the decline in infection level is slow. However over the same period there was no change in the prevalence of mastitis pathogens as no controls measures were adopted in India. In contrast to our study, Sumathi et al. [14], Dubal et al. [15], Ranjan et al. [16], Harini and Sumathi [17], Kurjogi and Kaliwal [18], AbdElrahman [19] and Jeykumar et al. [20] observed E. coli being second in prevalence following staphylococci. However, Marimuthu et al. [21] found 21\% of organisms isolated to be Bacillus spp. which was totally absent in our study and other studies mentioned earlier. Isolation of $E$. coli is indicative of poor hygienic conditions of animal environment as E. coli infects the udder via teat canal from the environment. Isolation of $C$. pyogenes is indicative of poor care and managemental practices. C. pyogenes alone establishes itself readily in mammary tissue causing subclinical mastitis. The situation worsens when $C$. pyogenes infect the udder along with other bacteria leading to chronic mastitis ultimate loss of the quarter.

\section{Antibiogram of S. aureus isolates}

Results of antibiotic sensitivity pattern for S. aureus isolates showed amikacin to be the most sensitive among all the antibiotics tested which was in accordance with Ranjan et al. [16]; higher than reported by Pankaj et al. [8], whereas on the lower side as compared with Kurjogi and Kaliwal [18]. However, S. aureus was the least sensitive against penicillin-G in our study that was similar to studies reported by Mohanty et al. [10] and Ranjan et al. [16] conducted around the country. The resistance of $S$. aureus to penicillin-G in our study may be attributed to the production of beta lactamase, an enzyme that inactivates penicillin and closely related antibiotics. The probable reason for the development of resistance by these organisms to penicillin- $G$ is conventional, prolonged and indiscriminate usage of this drug in the field conditions. The reasons for higher sensitivity against penicillin-G reported by Pankaj et al. [8] and Charaya et al. [9] might be that both these studies were conducted at organised farm where rational use of antibiotics is done.

\section{Antibiogram of streptococci}

Chloramphenicol has been found most sensitive (97\%) towards Streptococcus isolates irrespective of the species. Antibiogram pattern reported by Pankaj et al. [8] revealed higher sensitivity towards penicillin-G, neomycin, cloxacillin, gentamicin, amoxicillin, ceftriaxone and cefoperazone. However, few studies $[8,16,18]$ showed lesser sensitivity of Streptococcus isolates towards penicillin-G, gentamicin, streptomycin, ampicillin and amikacin as compared to present study. While comparing sensitivity of enrofloxacin, we found Ranjan et al. [16] and Mohanty et al. [10] to be on higher side whereas Pankaj et al. [8] and Abd-Elrahman [19] on lower side.

\section{Antibiogram of E. coli isolates}

$E$. coli is a major pathogen responsible for the causation of coliform mastitis that is an acute and potentially lethal type of mastitis. Selection of suitable antimicrobial for acute E. coli mastitis depends primarily on the sensitivity of the organism to the selected drug and the ability to maintain an effective concentration in plasma. Out of antibiotics tested chloramphenicol outstand with sensitivity of $94.59 \%$. Similar pattern were observed by Dubal et al. [15], Ranjan et al. [16], Charaya et al. [9] and Mohanty 
et al. [10] while Kurjogi and Kaliwal [18] and Abd-Elrahman [19] reported it to be on lower side. However, the least sensitivity was shown towards penicillin-G (13.51\%) that was in accordance with report of Mohanty et al. [10]. For successful treatment of coliform mastitis, along with suitable antimicrobial therapy, fluids, calcium, bicarbonates, antihistamines are suggested. However, the efficacy of therapy, particularly for antimicrobials, is unproven in coliform mastitis.

\section{Antibiogram of C. pyogenes isolates}

In our study more than $80 \%$ C. pyogenes isolates revealed sensitivity towards all the antibiotics except penicillin-G. Similar to our study, Dutta et al. [22] also recorded high sensitivity towards ampicillin but contrary to our study they found less sensitivity towards cloxacillin.

\section{Conclusion}

In conclusion, the present study indicated considerable prevalence of the disease and pathogens from clinical mastitis in Hisar and its surroundings. Appropriate treatment and control strategies should be formulated to eradicate or reduce major pathogens $S$. aureus, S. dysgalactiae, S. agalactiae and $E$. coli. Mastitis is the single largest cause of antimicrobial use in dairy farms. Extensive and indiscriminate use of antibiotics against these bacteria results into emergence of drug resistant mutants which cause treatment failure in animals have also become a potential health risk for humans, as they are able to cause direct and indirect transmission of infection. Therefore, establishing an antibiogram of pathogens is very important from the clinical and economic points of view. Therefore, dairy farm owners need to be advised to get antimicrobial susceptibility testing prior to the institution of proper line of treatment.

\section{Authors' Contributions}

GC, AS and MS planned the study design. GC and MS carried out the examination part. Analysis of the result was made by AS. GC and MS prepared the manuscript. AS, AK and PG finalized the manuscript. All authors read and approved the final manuscript.

\section{Acknowledgments}

The authors are highly thankful to Dean, College of Veterinary Sciences, LUVAS, Hisar for providing financial assistance and necessary facilities to carry out this work. The technical help rendered by Sh. Randhir Singh, Lab. Assistant, COVS, LUVAS, Hisar is gratefully acknowledged.

\section{Competing I nterests}

The authors declare that they have no competing interests.

\section{References}

1. Tanwar, P.S., Kumar, Y. and Sankhala, G. (2012) Economics of milk production among member and non-member of dairy cooperatives in Jaipur (Rajasthan). Indian J. Dairy Sci., 65(5): 2812-2820.

2. Dua, K. (2001) Studies on the incidence, etiology and estimated economic losses due to mastitis in Punjab and India - An update. Indian Dairyman, 53: 41-48.

3. Bansal, B.K. and Gupta, D.K. (2009) Economic analysis of bovine mastitis in India and Punjab - A review. Indian $J$. Dairy Sci., 67: 337-345.

4. Bhuvana, M. and Shome, B.R. (2013) Etiology of bovine mastitis. Proceedings of Model Training Course on "Bovine Mastitis: Theoretical and Practical Consideration in Management," p7-10.

5. Hogan, J.S., Gonzalez, R.N., Harmon, R.J., Nickerson, S.C., Oliver, S.P., Pankey, J.W. and Smith, K.L. (1999) Laboratory Handbook on Bovine Mastitis. Revised Edition, National Mastitis Council, Madison, WI.

6. Bauer, A.W., Kirby, W.W.M., Sherris, J.C. and Turck, M. (1966) Antibiotic susceptibility testing by a standardized single disc method. Am. J. Clin. Pathol., 45: 493.

7. Elango, A., Doraisamy, K.A., Rajarajan, G. and Kumaresan, G. (2010) Bacteriology of sub clinical mastitis and antibiogram of isolates recovered from cross bred cows. Indian J. Anim. Res., 44(4): 280-284.

8. Pankaj, Sharma, A., Chhabra. R. and Sindhu, N. (2012) Prevalence of sub clinical mastitis in cows: Its etiology and antibiogram. Indian J. Anim. Res., 46(4): 348-353.

9. Charaya, G.C., Sharma, A., Singh, M., Tiwari, S., Pankaj and Kumar, A. (2013) Subclinical mastitis at an organised farm: prevalence, etiology and antibiogram. Haryana Vet., 52: 30-32.

10. Mohanty, N.N., Das, P., Pany, S.S., Sarangi, L.N., Ranabijuli, S. and Panda, H.K. (2013) Isolation and antibiogram of Staphylococcus, Streptococcus and Escherichia coli isolates from clinical and subclinical cases of bovine mastitis. Vet. World, 6(10): 739-743.

11. Saidi, R., Khelef, D. and Kaidi, R. (2013) Bovine mastitis: Prevalence of bacterial pathogens and evaluation of early screening test. Afr. J. Microbiol. Res., 7(9): 777-782.

12. Tesfaye, A., Yohannes, A., Hunde, A., Tezera, T. and G/Tsadik, Z. (2013) Mastitis: Prevalence, risk factors and antimicrobial sensitivity patterns of bacterial isolates in dairy cattle at Holeta farm in Ethiopia. Afr. J. Agric. Res., 8(23): 2837-2842.

13. Alemu, G., Almaw, G. and Abera, M. (2014) Incidence rate of Staphylococcus aureus and Streptococcus agalactiae in subclinical mastitis at smallholder dairy cattle farms in Hawassa, Ethiopia. Afr. J. Microbiol. Res., 8(3): 252-256.

14. Sumathi, B.R., Veeregowda, B.M. and Gomes, A.R. (2008) Prevalence and antibiogram profile of bacterial isolates from clinical bovine mastitis. Vet. World, 1(8): 237-238.

15. Dubal, Z.B., Rahman, H., Pal, P., Kumar, A. and Pradhan, K. (2010) Characterization and antimicrobial sensitivity of the pathogens isolated from bovine mastitis with special reference to Escherichia coli and Staphylococcus spp. Indian $J$. Anim. Sci., 80(12): 1163-1167.

16. Ranjan, R., Gupta, M.K., Singh, S. and Kumar, S. (2010) Current trend of drug sensitivity in bovine mastitis. Vet. World, 3(1): 17-20.

17. Harini, H. and Sumathi, B.R. (2011) Screening of bovine milk samples for sub-clinical mastitis and antibiogram of bacterial isolates. Vet. World, 4: 358-359.

18. Kurjogi, M.M. and Kaliwal, B.B. (2011) Prevalence and antimicrobial susceptibility of bacteria isolated from bovine mastitis. Adv. Appl. Sci. Res., 2(6): 229-235.

19. Abd-Elrahman, A.H. (2013) Mastitis in housed dairy buffaloes: Incidence, etiology, clinical findings, antimicrobial sensitivity and different medical treatment against $E$. coli 
mastitis. Life Sci. j., 10(1): 532-538.

20. Jeykumar, M., Vinodkumar, G., Bashir, B.P. and Krovvidi, S. (2013) Antibiogram of mastitis pathogens in the milk of crossbred cows in Namakkal district, Tamil Nadu. Vet. World, 6: 354-356.

21. Marimuthu, M., Abdullah, M., Jesse, F.F., Mohammed, K., Adamu, L., Osman, A.Y., Abba, Y. and Tijjani, A. (2014)
Prevalence and antimicrobial resistance assessment of subclinical mastitis in milk samples from selected dairy farms. Am. J. Anim. Vet. Sci., 9(1): 65-70.

22. Dutta, T.K., Kumar, V.S.S. and Kotwal, S.K. (2007) Prevalence and antibiotic resistance pattern of bacteria from clinical and subclinical cases of bovine mastitis in Jammu region. Indian J. Anim. Sci., 77(6): 427-429.

$* * * * * * * *$ 